\title{
Nonsuperfluid Origin of the Nonclassical Rotational Inertia in a Bulk Sample of Solid ${ }^{4} \mathrm{He}$
}

\author{
John D. Reppy* \\ Laboratory of Atomic and Solid State Physics, Cornell University, Ithaca, New York 14853-2501, USA
}

(Received 13 April 2010; revised manuscript received 11 May 2010; published 21 June 2010)

\begin{abstract}
The torsional oscillator experiments described here examine the effect of disorder on the nonclassical rotational inertia (NCRI) of a solid ${ }^{4} \mathrm{He}$ sample. The NCRI increases with increasing disorder, but the period changes responsible for this increase occur primarily at higher temperatures. Contrary to expectations based on a supersolid scenario, the oscillator period remains relatively unaffected at the lowest temperatures. This result points to a nonsuperfluid origin for the NCRI.
\end{abstract}

DOI: 10.1103/PhysRevLett.104.255301

PACS numbers: 67.80.bd, 66.30.Ma

The work reported here investigates the changes in the period of a torsional oscillator (TO) containing a solid hcp ${ }^{4} \mathrm{He}$ sample as a result of plastic deformation of the sample. In the pioneering experiments of Kim and Chan [1,2] (KC) at Penn State, a decrease in the period of a TO containing a solid ${ }^{4} \mathrm{He}$ sample was observed for temperatures below $250 \mathrm{mK}$. KC interpreted the period decrease in terms of an onset of the growth of nonclassical rotational inertia (NCRI) [3] arising from a superfluidlike decoupling of a fraction of the solid ${ }^{4} \mathrm{He}$ moment of inertia from the TO. In subsequent work, Kim et al. [4] showed that the magnitude of the nonclassical rotational inertia fraction (NCRIF) is sensitive to ${ }^{3} \mathrm{He}$ impurity concentrations. The Penn State group also found a sensitivity to the method of sample preparation [5]. The experiments of Rittner and Reppy [6] demonstrated that annealing of the solid ${ }^{4} \mathrm{He}$ samples can greatly reduce the magnitude of the NCRI signal, suggesting that the level of disorder in the sample plays an important role in this phenomenon.

In a series of interesting experiments, Day and Beamish [7] (DB) have studied the shear modulus, $\mu$, of solid ${ }^{4} \mathrm{He}$. They find an anomalous increase, $\Delta \mu$, in the shear modulus at low temperatures which bears a striking resemblance to the temperature dependence of the NCRI signals observed in the TO experiments. DB argue that the temperature dependence of the shear modulus and the related phenomena can be understood in terms of the pinning of dislocation lines by ${ }^{3} \mathrm{He}$ as the temperature is lowered. Although it is tempting to attempt to explain the TO results in terms of the changes in the shear modulus, calculation [8] shows that the $10 \%$ to $20 \%$ variations seen in the modulus by DB are far too small to explain the TO results. West et al. [9] also report an increase in the shear modulus for hcp ${ }^{3} \mathrm{He}$. However, the NCRI signature is absent in ${ }^{3} \mathrm{He}$ experiments, which suggests a role for quantum statistics in these experiments.

The aim of the current experiment was to determine the influence on the NCRI of disorder induced by the plastic deformation of a solid ${ }^{4} \mathrm{He}$ TO sample. Plastic deformation of solid ${ }^{4} \mathrm{He}$ is known to result in the creation of dense populations of dislocations in the solid [10-13]. The de- formation of the solid and the accompanying generation of a high-density population of dislocations was expected to lead to an increase in the NCRI signal, as has been confirmed by this experiment.

The TO cell used for the current experiments is shown in Fig. 1. At low temperatures the frequency of the TO is $818 \mathrm{~Hz}$ and the rim velocity is in the range of 10 to $20 \mu \mathrm{m} / \mathrm{s}$. An inner cylinder, attached to the flexible diaphragm at the top of the cell, defines an annular region containing the solid ${ }^{4} \mathrm{He}$ sample. It has a height of $0.917 \mathrm{~cm}$, a radial gap of $0.032 \mathrm{~cm}$, and a mean radius of $0.714 \mathrm{~cm}$. The gaps at the top and bottom of the inner cylinder are $0.025 \mathrm{~cm}$. The TO period at liquid helium temperatures is $1.219 \mathrm{~ms}$ and the total period shift for a solid sample with a density of $0.200 \mathrm{gm} / \mathrm{cm}^{3}$ is calculated to be $185 \mathrm{~ns}$. A Straty-Adams (SA) capacitance gauge [14] with mbar pressure sensitivity is mounted on the upper side of the diaphragm and the region surrounding the gauge is enclosed, with a fill line added to allow the volume above

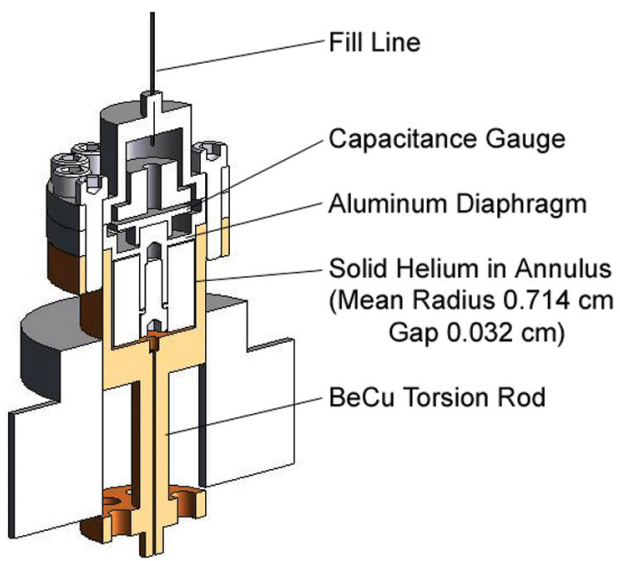

FIG. 1 (color). The torsional oscillator, consisting of three sections, is shown in cross section. The body and torsion rod are made from a single piece of hardened $\mathrm{BeCu}$ alloy. The other sections, including the flexible diaphragm, were machined from a high strength aluminum alloy. The Straty-Adams gauge electrodes are brass, and the upper fill line consists of a section of CuNi tubing with an internal diameter of $0.01 \mathrm{~cm}$. 
the diaphragm to be filled with liquid ${ }^{4} \mathrm{He}$. Hydrostatic pressure can then be applied to the diaphragm to produce a controlled displacement of the diaphragm and the attached inner cylinder. More details of this technique are given in Rittner et al. [15]. The displacement is monitored by the capacitance of the SA gauge, suitably corrected for the pressure-dependent dielectric constant of the liquid helium between the plate capacitor plates. Since the TO has a pressure sensitivity of $0.88 \mathrm{~ns} / \mathrm{bar}$, the sample pressure is monitored with the SA gauge to insure that period shifts observed in the course of the experiment are not due to pressure changes within the cell.

The maximum pressure that can be applied to the diaphragm is limited to the melting pressure of ${ }^{4} \mathrm{He}$ at low temperature. At this pressure, the inner cylinder is displaced by $1.9 \mu \mathrm{m}$ relative to the outer wall of the cell, resulting in a shear strain in the annulus of $5.9 \times 10^{-3}$. This level of strain is well above the threshold for the plastic deformation of hop ${ }^{4} \mathrm{He}$ [7]. The solid ${ }^{4} \mathrm{He}$ samples are formed by the blocked capillary (BC) method, using standard ${ }^{4} \mathrm{He}\left(0.3 \mathrm{ppm}{ }^{3} \mathrm{He}\right.$ impurity). The cell is initially filled with liquid to a pressure near $60 \mathrm{bar}$, then cooled to freeze the sample. At the completion of freezing the sample pressure has been reduced to about 40 bar. Sample formation by the $\mathrm{BC}$ technique results in a polycrystalline sample with a relatively high degree of initial disorder. In an attempt to reduce the initial degree of disorder, most samples are annealed for up to $20 \mathrm{~h}$ at temperatures above $1 \mathrm{~K}$. In Fig. 2 the period data are shown for a ${ }^{4} \mathrm{He}$ sample with two different levels of disorder. Period data for the empty cell, shifted to match the other data sets at $20 \mathrm{mK}$, are also shown. The magnitude of the NCRI signal, is determined in the usual way by subtracting the raw period

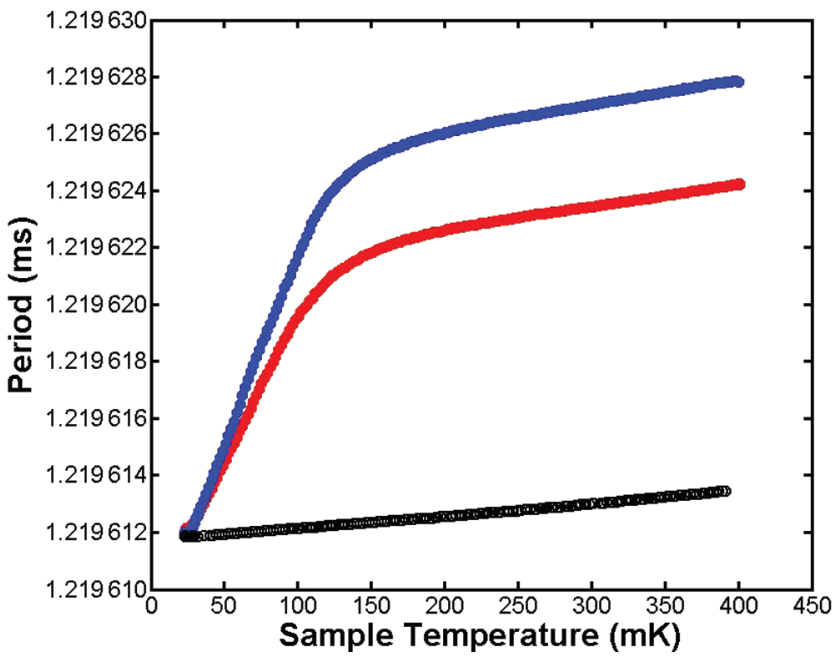

FIG. 2 (color). Period data for the initial sample of bulk solid ${ }^{4} \mathrm{He}$ are shown in red as a function of temperature. The period data obtained following the deformation of the sample by an application of 10.3 bar to the flexible diaphragm are plotted in blue. The bottom data, plotted in black, are the period data for the empty cell shifted upward by $110 \mathrm{~ns}$. data from a linear "background" function determined by a fit to the period data above the onset temperature. The initial sample, middle data set in Fig. 2, was cooled to $20 \mathrm{mK}$ after being annealed at $1.6 \mathrm{~K}$. The magnitude of the NCRI for this sample was $9.1 \mathrm{~ns}$ for a $4.9 \%$ NCRIF. The temperature was raised then to $250 \mathrm{mK}$, and ${ }^{4} \mathrm{He}$ was slowly condensed into the volume above the diaphragm. When this volume was full, the pressure was raised to $10.3 \mathrm{bar}$, to produce a $0.81 \mu \mathrm{m}$ displacement of the inner cylinder and a shear strain of $2.55 \times 10^{-3}$. The pressure was then reduced to near zero by pumping on the fill line, thus returning the diaphragm and inner cylinder to their original positions. The upper data set was obtained following the deformation of the sample. The NCRI has now increased to $12.6 \mathrm{~ns}$ or a $6.8 \%$ NCRIF. There are two important features: (i) the TO period is increased over a wide temperature range above the "supersolid" onset; (ii) the increase in the period approaches zero as the temperature is reduced to $20 \mathrm{mK}$.

The effect of the deformation on the TO period is most clearly displayed by subtracting the period data for the undeformed sample from the period values after deformation. These data are shown in Fig. 3. These data present a remarkable contrast to expectations based on a scenario of a superfluidlike decoupling of a fraction of the solid moment of inertia. In this scenario, the sample is assumed to be firmly locked to the oscillator above the NCRI onset temperature, and it should be unaffected by the degree of disorder. Thus, the major changes in period, reflecting the growth of the NCRI signal, would occur at temperatures below the onset, rather than at high temperatures as seen in this experiment.

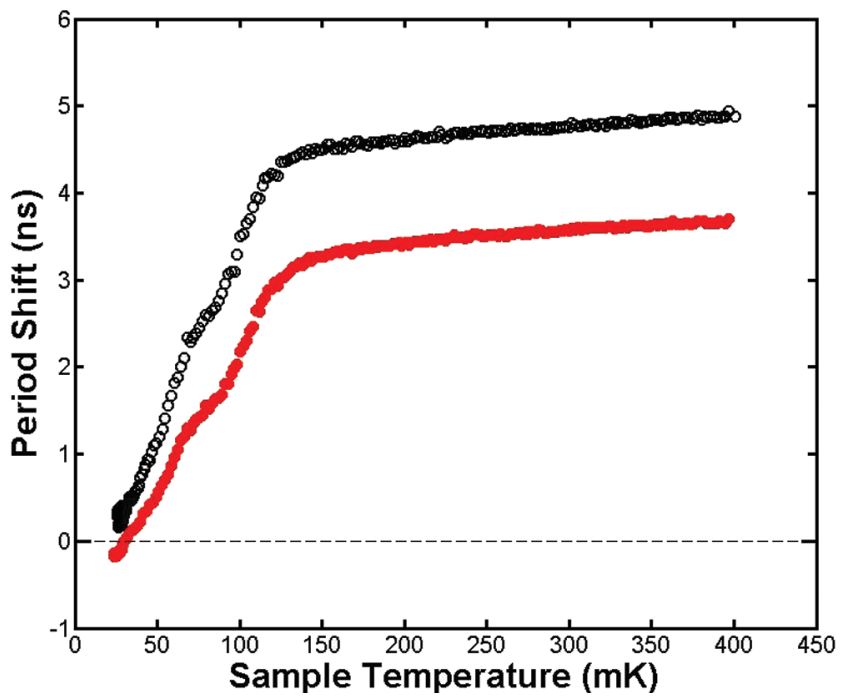

FIG. 3 (color). Data for the period shift between the period following deformation and the period of the initial sample are plotted as functions of the temperature. The lower data set, in red, was obtained after a pressure of 10.3 bar had been applied to the flexible diaphragm, while the second set with the larger period increase was obtained after the application of 13.8 bar. 
The data for the total dissipation, $Q^{-1}$ total, for the TO corresponding to the period data of Fig. 2 are shown in Fig. 4 in an unshifted form. The total dissipation can be expressed as the sum of the individual contributions from the empty cell dissipation and dissipation due to the solid, $Q^{-1}{ }_{\text {total }}=Q^{-1}{ }_{\text {empty cell }}+Q^{-1}{ }_{\text {solid }}$. At the lowest temperature, the dissipation of the empty cell accounts for the entire TO dissipation, with no additional contribution from the solid sample. As the temperature is increased, the solid contribution to the dissipation grows and passes through a peak in the neighborhood of $100 \mathrm{mK}$. As the temperature is raised above onset, the solid continues to make a substantial contribution to the total dissipation of the TO above the NCRI onset. Again, this behavior for the dissipation is contrary to what would be expected in the supersolid scenario, where the solid is locked to the TO and should contribute little if any additional dissipation. The dissipative dynamics of the solid ${ }^{4} \mathrm{He}$ system have been extensively studied with the TO technique; for a recent work see Hunt et al. [16].

The effects of annealing were investigated in a sample that had been subject to the maximum strain. An initial annealing of this sample took place at a temperature of $0.820 \mathrm{~K}$ for a period of $19 \mathrm{~h}$. During this time the period decreased 0.6 ns. Following this anneal, the temperature was lowered to $20 \mathrm{mK}$. The data obtained while warming from this temperature to $1.2 \mathrm{~K}$ are shown in Fig. 5. The sample was further annealed at $1.2 \mathrm{~K}$. Raising the temperature to $1.4 \mathrm{~K}$ resulted in an almost discontinuous decrease in the TO period. Finally, the sample was held at $1.6 \mathrm{~K}$ for a period of $4 \mathrm{hrs}$ without any further obvious relaxation in the period. The sample was then cooled again to $20 \mathrm{mK}$. At the lowest temperature the period of the

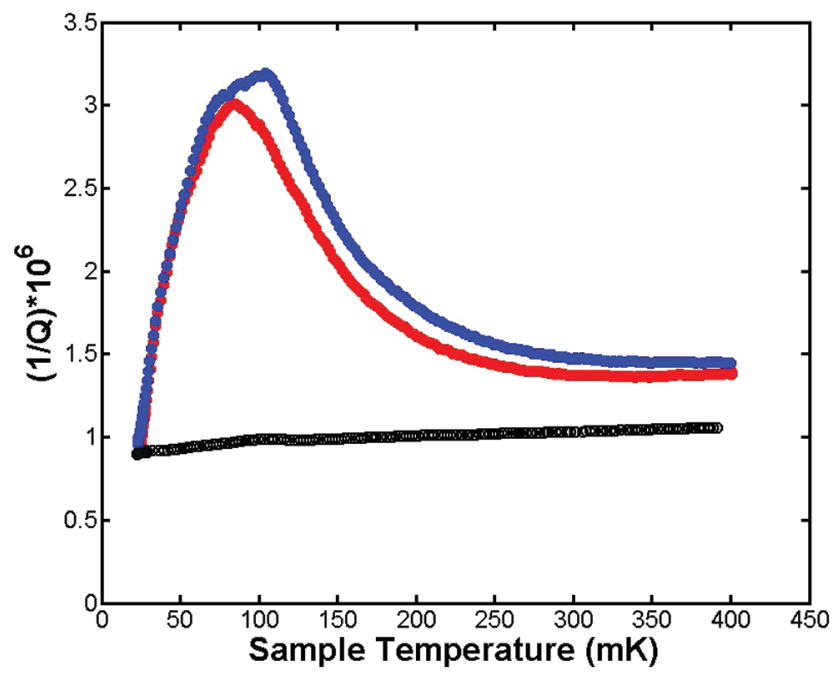

FIG. 4 (color). Dissipation data for the empty cell are plotted in black, the initial sample in red, and the data for the sample after deformation in blue. Note that the dissipation data for the empty cell have not been shifted. At the lowest temperature all three data sets have the same dissipation. annealed sample returned to within $1 \mathrm{~ns}$ of the preannealed value. In contrast, at $400 \mathrm{mK}$ the period difference between the preannealed sample and the final annealed sample was $11 \mathrm{~ns}$. Although, the dislocation lines introduced at $250 \mathrm{mK}$ by plastic deformation can be readily removed by annealing at temperatures above $1 \mathrm{~K}$, it is also clear that there are other forms of disorder in the sample that are less susceptible to annealing; it is interesting to note, however, that the influence of the remaining unannealed disorder freezes out over much same temperature range, below $0.2 \mathrm{~K}$, as does that of the induced dislocation lines.

The most important conclusions to be drawn from this experiment are that the high temperature period of a TO containing a solid ${ }^{4} \mathrm{He}$ sample is strongly dependent on the level of disorder in the sample, with an increase in the TO period following an increase in disorder. The period increase and the additional dissipation associated with sample disorder both vanish at the lowest temperature. An analogous low temperature behavior for the shear modulus has been reported by Day, Syschenko, and Beamish [17]. They find the low temperature value of the shear modulus to be relatively unaffected by additional disorder or annealing of the sample; however, several other aspects of their work are not in accord with the present experiment.

Since the original KC experiments, a number of models based on nonsuperfluid mechanisms have been developed to explain the NCRI phenomenon. Among these are the suggestion that a glass transition may take place in the solid [18], the two-level system model of Andreev [19], as well as a dislocation line model, developed by Iwasa [20].

In the case of an ordinary solid, the sample will be accelerated by an elastic interaction with the walls. When the ratio between the mean radius of the annulus and the radial gap, $\Delta R / R$, is large, as for the present $\mathrm{TO}$, the annular geometry can be approximated by parallel plates

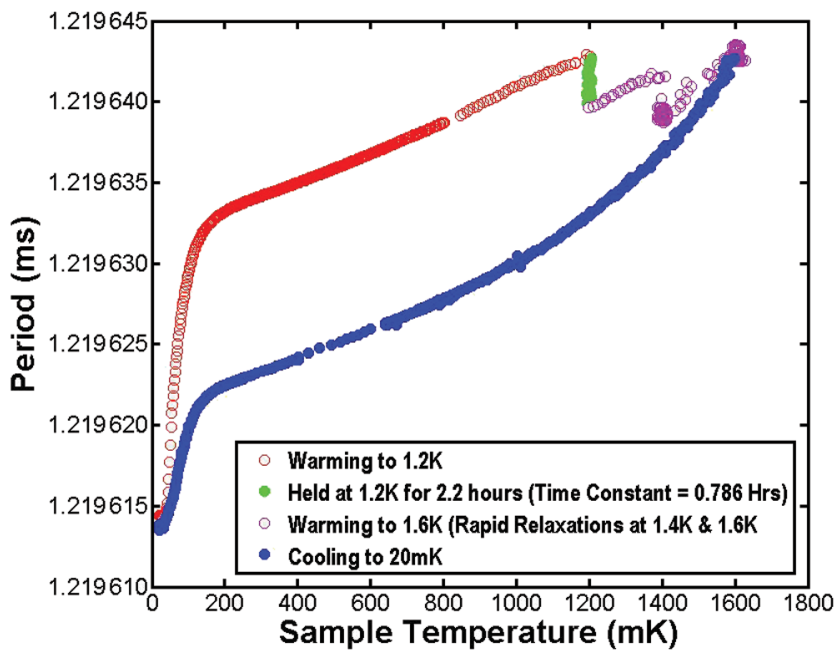

FIG. 5 (color). Period data for a series of temperature sweeps, including high temperature annealing, are shown as a function of temperature. 
separated by $\Delta R$. The displacement of the plates will be $R \theta_{0} \sin (\omega, t)$, where $\theta_{0}$ is the maximum angular displacement of the TO. The motion of the solid is governed by a wave equation, with a solution for the angular displacement of the solid, $\theta(x, t)=\theta_{0} \sin (\omega t) \times$ $[\cos (2 \pi \Delta R x / \lambda) / \cos (\Delta R / \lambda)]$, where $\lambda=(2 \pi / \omega) \times$ $(\mu / \rho)^{1 / 2}$ is a characteristic wavelength and $\mu$ is an effective shear modulus of the disordered solid. The $x$ axis, normal to the surface of the plates, has its origin at the midpoint between the plates. The mean angular displacement of the solid, $\left(\theta_{0}+\Delta \theta\right) \sin (\omega t)=\theta_{0}[\lambda /(\pi \Delta R)] \times$ $\tan (\pi \Delta R / \lambda) \sin (\omega t)$ and is slightly larger by the amount, $\Delta \theta$, than $\theta_{0} ;\left(\Delta \theta / \theta_{0}\right)=[[\lambda /(\pi \Delta R)] \tan (\pi \Delta R / \lambda)-1]$. Expanding $\tan (\pi \Delta R / \lambda)$ to second order in $(\pi \Delta R / \lambda)$, gives $\left(\Delta \theta / \theta_{0}\right) \cong \rho\left(\Delta R^{2} \omega^{2} / 12 \mu\right.$, or $\mu \cong \rho\left(\Delta R^{2} \omega^{2}\right)$ $\left.\left(12 \Delta \theta / \theta_{0}\right)\right)$. The $\omega^{2}$ dependence might be tested with a double frequency TO.

Neglecting frictional terms, the total torque acting on the $\mathrm{TO}$ is $\tau_{\mathrm{TO}}=-k \theta(t)+\tau_{s}=-I_{\mathrm{TO}} \omega^{2} \theta_{0} \sin (\omega t)$, where $\tau_{s}=I_{s} \omega^{2}\left(\theta_{0}+\Delta \theta\right) \sin (\omega t)$ is the reaction torque due to the solid. $I_{\mathrm{TO}}$ and $I_{S}$ are the moments of inertia of the empty TO and the solid sample, respectively, and $k$ is the torsion constant. Solving the torque equation for $\omega=$ $2 \pi / P$, where $P$ is the oscillator period, we have $P=$ $2 \pi \sqrt{\left[I_{\mathrm{TO}}+I_{S}\left(1+\left(\Delta \theta / \theta_{0}\right)\right)\right] / k}$. In the standard supersolid picture, the expression for the TO period is $P=$ $2 \pi \sqrt{\left[I_{\mathrm{TO}}+I_{S}\left(1-\left(\rho_{S} / \rho\right)\right)\right] / k}$. Thus a decrease in $\left(\Delta \theta / \theta_{0}\right)$, which might occur as a result of an increase in the shear modulus. will be reflected by a decrease in the TO period that would be interpreted as an increase in $\left(\rho_{S} / \rho\right)$ or the NCRIF in the supersolid scenario.

Iwasa [20] has suggested that under a shear stress, $\sigma$, there will be an additional displacement strain due to dislocation line motion, $\epsilon=\left[(\Omega \Lambda) /\left(\pi \rho \omega_{0}^{2}\right)\right] \sigma$, where $\Lambda$ is the line density, $\Omega$ is an orientation factor, and $\omega_{0}$ is the resonant frequency for a pinned dislocation line. This equation serves to define an effective shear modulus $\mu^{\prime}=$ $\left(\pi \rho \omega_{0}^{2}\right) /(\Omega \Lambda)$. Substituting this value for $\mu$ in the previous expression for $\left(\Delta \theta / \theta_{0}\right)$, gives $\left(\Delta \theta / \theta_{0}\right)=$ $\left(\Omega \Lambda \Delta R^{2} \omega^{2}\right) /\left(12 \pi \omega_{0}^{2}\right)$. The frequency of pinned lines, $\omega_{0}$, depends on the length of line between pinning centers that will, in turn, will depend on details of the sample. As an example, taking $5 \mu \mathrm{m}$ as the separation between pinning centers, $\omega_{0}=1.6 \times 10^{7} \mathrm{~Hz}$. A $1 \%$ shift in $\left(\rho_{S} / \rho\right)$, or $\left(\Delta \theta / \theta_{0}\right)=10^{-2}$ would require $\Omega \Lambda=2.3 \times$ $10^{10}$ lines $/ \mathrm{cm}^{2}$. This value for the line density is several orders of magnitude larger than that observed in ultrasonic experiments [10-13]. In the Iwasa model, the decrease in period at low temperatures results from additional pinning by ${ }^{3} \mathrm{He}$ atoms that condense on the lines as the temperature is lowered. Shorter lines will have higher resonant frequencies and a reduced $\Delta \theta / \theta_{0}$. Above approximately $200 \mathrm{mK}$, depending on the ${ }^{3} \mathrm{He}$ concentration in the sample, the ${ }^{3} \mathrm{He}$ atoms will evaporate and the TO period will become relatively independent of temperature.

Iwasa's model has attractive features, however, the large value required for the line density may be a problem; it is also difficult to see how dislocation line motion might account for the observed NCRI in the case of solid ${ }^{4} \mathrm{He}$ contained in porous Vycor glass [1] where the line length would be restricted to the pore size of $10 \mathrm{~nm}$. In addition, a model based solely on dislocation line dynamics does not incorporate the effects of quantum statistics that may be required to explain the absence of an observable NCRI in hcp ${ }^{3} \mathrm{He}[9]$.

The author thanks Xiao Mi for his assistance, and also thanks J. V. Reppy and E.N. Smith. He acknowledges profitable discussions with E. Mueller, N. Ashcroft, and V. Ambegaokar, as well as A. Balatsky, M. Graf, and M. Chan. This work has been supported by the National Science Foundation Grant No. DMR-0605864 and the CCMR Grant No. DMR-0520404.

*jdr13@cornell.edu

[1] E. Kim and M.H.W. Chan, Nature (London) 427, 225 (2004).

[2] E. Kim and M. H.W. Chan, Science 305, 1941 (2004).

[3] A. J. Leggett, Phys. Rev. Lett. 25, 1543 (1970).

[4] E. Kim, J.S. Xia, J. T. West, X. Lin, A. C. Clark, and M. H. W. Chan, Phys. Rev. Lett. 100, 065301 (2008).

[5] A. C. Clark, J. T. West, and M.H. W. Chan, Phys. Rev. Lett. 99, 135302 (2007).

[6] Ann Sophie C. Rittner and J. D. Reppy, Phys. Rev. Lett. 97, 165301 (2006).

[7] J. Day and J.R. Beamish, Nature (London) 450, 853 (2007).

[8] A. C. Clark, J. D. Maynard, and M. H. W. Chan, Phys. Lett. B 77, 155301 (2008).

[9] J. T. West, O. Syshchenko, J. Beamish, and M. H. W. Chan, Nature Phys. 5, 598 (2009).

[10] H. Suzuki, J. Phys. Soc. Jpn. 35, 1472 (1973).

[11] D. J. Sanders, H. Kwun, A. Hikata, and C. Elbaum, Phys. Rev. Lett. 39, 815 (1977).

[12] F. Tsuruoka and Y. Hiki, Phys. Rev. B 20, 2702 (1979).

[13] I. Iwasa, A. Araki, and H. Suzuki, J. Phys. Soc. Jpn. 46, 1119 (1979).

[14] G. C. Straty and E. D. Adams, Rev. Sci. Instrum. 40, 1393 (1969).

[15] Ann Sophie C. Rittner, W. Choi, E. J. Mueller, and J. D. Reppy, Phys. Rev. B 80, 224516 (2009).

[16] B. Hunt, E. Pratt, V. Gadagkar, M. Yamashita, A. V. Balatsky, and J. C. Davis, Science 324, 632 (2009).

[17] J. Day, O. Syshchenko, and J. Beamish, Phys. Rev. B 79, 214524 (2009).

[18] Z. Nussinov, A. V. Balatsky, M. J. Graf, and S. A. Trugman, Phys. Rev. B 76, 014530 (2007).

[19] A. F. Andreev, JETP Lett. 85, 585 (2007).

[20] I. Iwasa, Phys. Rev. B 81, 104527 (2010). 